\title{
On the Weyl-Ahlfors theory of derived curves
}

\author{
Dinh Tuan Huynh, Song-Yan Xie*
}

\begin{abstract}
For derived curves intersecting a family of decomposable hyperplanes in subgeneral position, we obtain an analog of the Cartan-Nochka Second Main Theorem, generalizing a classical result of Fujimoto about decomposable hyperplanes in general position.
\end{abstract}

Keywords: Value distribution theory, Second Main Theorem, entire curves, Nochka weights, defect relation, subgeneral position, Wronskians

Mathematics Subject Classification 2010: 32H30, 32A22

\section{Introduction}

Value distribution theory was started by Nevanlinna [7] by relating the intersection frequency of a holomorphic map $f: \mathbb{C} \rightarrow \mathbb{P}^{1}(\mathbb{C})$ with $q \geq 3$ distinct points in $\mathbb{P}^{1}(\mathbb{C})$, and the growth rate of $f$. This quantifies the classical little Picard theorem, and also generalizes the fundamental theorem of algebra from polynomials to meromorphic functions.

In higher dimension, Cartan [2] explored Nevanlinna theory in the setting of a linearly nondegenerate entire curve $f: \mathbb{C} \rightarrow \mathbb{P}^{n}(\mathbb{C})$ together with a family of $q \geq n+2$ hyperplanes $\left\{H_{i}\right\}_{i=1, \ldots, q}$ in general position, and he obtained a second main theorem:

$$
(q-n-1) T_{f}(r) \leq \sum_{i=1}^{q} N_{f}^{[n]}\left(r, H_{i}\right)+S_{f}(r),
$$

(see section 2 for meanings of these notations) by introducing a Wronskian technique, which is indispensable in the subject [10, 11]. For hyperplanes $\left\{H_{i}\right\}_{i=1, \ldots, q}$ in $N$-subgeneral position, i.e., there exists some embedding $\mathbb{P}^{n}(\mathbb{C}) \hookrightarrow \mathbb{P}^{N}(\mathbb{C})$ such that $\left\{H_{i}=H_{i}^{\prime} \cap \mathbb{P}^{n}(\mathbb{C})\right\}_{i=1, \ldots, q}$ are the restrictions of hyperplanes $\left\{H_{i}^{\prime} \subset \mathbb{P}^{N}(\mathbb{C})\right\}_{i=1, \ldots, q}$ in general position, Cartan anticipated that there shall be

$$
(q-2 N+n-1) T_{f}(r) \leq \sum_{i=1}^{q} N_{f}^{[n]}\left(r, H_{i}\right)+S_{f}(r),
$$

and this conjecture was proved by Nochka [8] by means of the so-called Nochka weights.

Meanwhile, independently, Weyl's [16, 15] restarted the study of value distribution of entire curves in $\mathbb{P}^{N}(\mathbb{C})$ with respect to high codimension projective subspaces, by introducing the associated derived curves which assign every point $f(z)$ with the osculating $k^{\text {th }}$-planes passing through that point (see Subsection 2.3). In the same vein, Ahlfors [1] successfully established a second main theorem type estimate for derived curves, which embraces the inequality (1) of Cartan when $k=0$

\footnotetext{
* partially supported by NSFC Grant No. 11688101
} 
and the targets are hyperplanes. The reader is referred to [17, 12] for expositions about Weyl-Ahlfors' theory.

Since then Weyl-Ahlfors theory has much progress. Notably, Stoll [13, 14] studied meromorphic maps from parabolic spaces to projective spaces; Cowen-Griffiths [4] gave a simplified proof of Ahlfors' result using negative curvature; Fujimoto [5, 6] established a second main theorem for derived curves of linearly nondegenerate entire curves with optimal truncation level; Chen [3] generalized the Ahlfors' result for degenerated entire curves.

Inspiring by the works [5, 3], it would be natural to seek a second main theorem for derived curves, having optimal truncation level, without assuming the nondegeneracy of the entire curves. Here is our result in this direction, which is a generalization of Cartan-Nochka's Second Main Theorem.

Theorem 1.1. Let $f: \mathbb{C} \rightarrow \mathbb{P}^{N}(\mathbb{C})$ be an entire holomorphic curve, and let $\mathbb{P}^{n}(\mathbb{C}) \subset \mathbb{P}^{N}(\mathbb{C})$ be the smallest linear projective subspace containing $f(\mathbb{C})$. For a fixed integer $k=0,1, \ldots, n$, let $A_{1}, \ldots, A_{q}$ be $q$ decomposable hyperplanes of $\mathbb{P}\left(\Lambda^{k+1}\left(\mathbb{C}^{N+1}\right)\right)$ in general position such that none of them contains the induced Plücker subset $\mathbb{P}\left(\Lambda^{k+1}\left(\mathbb{C}^{n+1}\right)\right) \subset \mathbb{P}\left(\Lambda^{k+1}\left(\mathbb{C}^{N+1}\right)\right)$. Then the $k$-th derived curve $F_{k}$ of $f$ satisfies

$$
\left(q-2\left(\begin{array}{c}
N+1 \\
k+1
\end{array}\right)+\left(\begin{array}{c}
n+1 \\
k+1
\end{array}\right)\right) T_{F_{k}}(r) \leq \sum_{i=1}^{q} N_{F_{k}}^{[(k+1)(n-k)]}\left(r, A_{i}\right)+S_{F_{k}}(r) .
$$

In fact, this result follows directly from the following stronger statement (see Remark 2.1).

Main Theorem. Let $f: \mathbb{C} \rightarrow \mathbb{P}^{n}(\mathbb{C})$ be a linearly nondegenerate entire holomorphic curve. For a fixed integer $k=0,1, \ldots, n$, let $A_{1}, \ldots, A_{q} \subset \mathbb{P}\left(\Lambda^{k+1}\left(\mathbb{C}^{n+1}\right)\right)$ be $q$ decomposable hyperplanes such that any $\mathfrak{N}$ of them have empty intersection. Then the $k$-th derived curve $F_{k}$ of $f$ satisfies

$$
(q-2 \mathfrak{N}+\mathfrak{n}) T_{F_{k}}(r) \leq \sum_{i=1}^{q} N_{F_{k}}^{[(k+1)(n-k)]}\left(r, A_{i}\right)+S_{F_{k}}(r)
$$

where $\mathfrak{n}:=\operatorname{dim}_{\mathbb{C}} \Lambda^{k+1}\left(\mathbb{C}^{n+1}\right)=\left(\begin{array}{c}n+1 \\ k+1\end{array}\right)$.

Terminologies and notation will be explained in Section 2, while a complete proof will be reached in Section 3, which depends on classical techniques of Cartan's Wronskian [2], Nochka's weight [8] and Fujimoto's vanishing order estimates [5]. Whence a defect relation (42) can be concluded in Section 4, Theorem 1.1 improves a previous result of Chen [3] by providing an effective truncation level $(k+1)(n-k)$, which is optimal as shown by an example of Fujimoto [5]. For $k=0$, we recover the celebrated Cartan-Nochka's Theorem [8]. When $\mathfrak{n}=\mathfrak{N}$, our defect relation (42) coincides with a result of Fujimoto [5] for decomposable hyperplanes in general position.

\section{Acknowledgments}

Both authors are grateful to the Academy of Mathematics and System Sciences in Beijing for nice working conditions. Huynh also wants to thank the support from Hue University.

\section{Preliminaries}

\subsection{Nevanlinna theory}

We denote by $\Delta_{r} \subset \mathbb{C}$ the disk of radius $r>0$ centered at the origin. Fix a truncation level $m \in$ $\mathbb{N} \cup\{\infty\}$, for an effective divisor $E=\sum_{i} \alpha_{i} a_{i}$ on $\mathbb{C}$ where $\alpha_{i} \geq 0, a_{i} \in \mathbb{C}$, the $m$-truncated degree 
of the divisor $E$ on the disk $\Delta_{r}$ is given by

$$
n^{[m]}(r, E):=\sum_{a_{i} \in \Delta_{r}} \min \left\{m, \alpha_{i}\right\}
$$

the truncated counting function at level $m$ of $E$ is then defined by taking the logarithmic average

$$
N^{[m]}(r, E):=\int_{1}^{r} \frac{n^{[m]}(t, E)}{t} \mathrm{~d} t
$$

When $m=\infty$, for abbreviation we write $n(t, E), N(r, E)$ for $n^{[\infty]}(t, E), N^{[\infty]}(r, E)$.

Let $f: \mathbb{C} \rightarrow \mathbb{P}^{n}(\mathbb{C})$ be an entire holomorphic curve having a reduced representation $f=\left[f_{0}\right.$ : $\left.\cdots: f_{n}\right]$ in the homogeneous coordinates $\left[z_{0}: \cdots: z_{n}\right]$ of $\mathbb{P}^{n}(\mathbb{C})$. Let $D=\{Q=0\}$ be a divisor in $\mathbb{P}^{n}(\mathbb{C})$ defined by a homogeneous polynomial $Q \in \mathbb{C}\left[z_{0}, \ldots, z_{n}\right]$ of degree $d \geq 1$. If $f(\mathbb{C}) \not \subset D$, then $f^{*} D=\sum_{a \in \mathbb{C}} \operatorname{ord}_{a} f^{*} Q$ is a divisor on $\mathbb{C}$. We then define the truncated counting function of $f$ with respect to $D$ as

$$
N_{f}^{[m]}(r, D):=N^{[m]}\left(r, f^{*} D\right)
$$

which measures the intersection frequency of $f(\mathbb{C})$ with $D$. If $f^{*} D=\sum_{i} \mu_{i} a_{i}$, where $\mu_{i}>0$ and $\mu=\min \left\{\mu_{i}\right\}$, then we say that $f$ is completely $\mu$-ramified over $D$, with the convention that $\mu=\infty$ if $f(\mathbb{C}) \cap \operatorname{supp} D=\emptyset$. Next, the proximity function of $f$ associated to the divisor $D$ is given by

$$
m_{f}(r, D):=\int_{0}^{2 \pi} \log \frac{\left\|f\left(r e^{i \theta}\right)\right\|_{\max }^{d}\|Q\|_{\max }}{\left|Q(f)\left(r e^{i \theta}\right)\right|} \frac{\mathrm{d} \theta}{2 \pi},
$$

where $\|Q\|_{\max }$ is the maximum absolute value of the coefficients of $Q$ and where

$$
\|f(z)\|_{\max }:=\max \left\{\left|f_{0}(z)\right|, \ldots,\left|f_{n}(z)\right|\right\} \text {. }
$$

Since $|Q(f)| \leq\left(\begin{array}{c}d+n \\ n\end{array}\right)\|Q\|_{\max } \cdot\|f\|_{\max }^{d}$, we see that $m_{f}(r, D) \geq O(1)$ is bounded from below by some constant. Lastly, the Cartan order function of $f$ is defined by

$$
T_{f}(r):=\frac{1}{2 \pi} \int_{0}^{2 \pi} \log \left\|f\left(r e^{i \theta}\right)\right\|_{\max } \mathrm{d} \theta .
$$

The Nevanlinna theory is then established by comparing the above three functions. It consists of two fundamental theorems (for a comprehensive exposition, see Noguchi-Winkelmann [9]).

First Main Theorem. Let $f: \mathbb{C} \rightarrow \mathbb{P}^{n}(\mathbb{C})$ be a holomorphic curve and let $D$ be a hypersurface of degree $d$ in $\mathbb{P}^{n}(\mathbb{C})$ such that $f(\mathbb{C}) \not \subset D$. Then one has the estimate

$$
m_{f}(r, D)+N_{f}(r, D)=d T_{f}(r)+O(1)
$$

for every $r>1$, whence

$$
N_{f}(r, D) \leq d T_{f}(r)+O(1)
$$

Hence the counting function is bounded from above by some multiple of the order function. The reverse direction is usually much harder, and one often needs to take the sum of the counting functions of many divisors. Such types of estimates are so-called second main theorems.

Throughout this paper, for an entire curve $f$, the notation $S_{f}(r)$ means a real function of $r \in \mathbb{R}^{+}$ such that

$$
S_{f}(r) \leq O\left(\log \left(T_{f}(r)\right)\right)+\epsilon \log r
$$

for every positive constant $\epsilon$ and every $r$ outside of a subset (depending on $\epsilon$ ) of finite Lebesgue measure of $\mathbb{R}^{+}$. In the case where $f$ is rational, we understand that $S_{f}(r)=O(1)$. In any case we always have

$$
\liminf _{r \rightarrow \infty} \frac{S_{f}(r)}{T_{f}(r)}=0
$$




\subsection{Grassmann algebra}

Let $E$ be a $\mathbb{C}$-vector space of dimension $M+1$. The graded exterior algebra $\Lambda^{\bullet} E=\oplus_{k=0}^{M} \Lambda^{k} E$, equipped with the exterior wedge product, is called a Grassmann algebra. Every element in $\Lambda^{k} E$ is called a $k$-vector, and it is said to be decomposable if it can be written neatly as $a_{1} \wedge \cdots \wedge a_{k}$ for some $k$ vectors $a_{1}, \ldots, a_{k} \in E$.

Given a basis $\left\{e_{0}, \ldots, e_{M}\right\}$ of $E$, then $\Lambda^{k+1} E$ has the basis $\left\{e_{i_{0}} \wedge \cdots \wedge e_{i_{k}}\right\}_{0 \leq i_{0}<i_{1}<\cdots<i_{k} \leq M}$. In this coordinate system, for $k+1$ vectors $a_{i}=\sum_{j=0}^{M} a_{i, j} e_{j}$ where $i=0, \ldots, k$, direct computation shows:

$$
a_{0} \wedge \cdots \wedge a_{k}=\sum_{0 \leq i_{0}<i_{1}<\cdots<i_{k} \leq M} a\left(i_{0}, \ldots, i_{k}\right) e_{i_{0}} \wedge \cdots \wedge e_{i_{k}}
$$

where $a\left(i_{0}, \ldots, i_{k}\right):=\operatorname{det}\left(\left(a_{\alpha, i_{\beta}}\right)_{0 \leq \alpha, \beta \leq k}\right)$.

\subsection{Derived curves}

Let $f: \mathbb{C} \rightarrow \mathbb{P}^{n}(\mathbb{C})$ be a linearly nondegenerate entire holomorphic curve with a reduced representation $f=\left[f_{0}: \cdots: f_{n}\right]$ in the homogeneous coordinates. Note that

$$
\tilde{f}:=\left(f_{0}, \ldots, f_{n}\right): \mathbb{C} \rightarrow \mathbb{C}^{n+1} \backslash\{0\}
$$

provides a lifting of $f$ along the natural projection $\pi: \mathbb{C}^{n+1} \backslash\{0\} \rightarrow \mathbb{P}^{n}(\mathbb{C})$. For $k=1, \ldots, n$, to construct a $k^{\text {th }}$-derived curve we first collect all the derivatives

$$
\tilde{f}^{(\ell)}=\left(f_{0}^{(\ell)}, \ldots, f_{n}^{(\ell)}\right): \mathbb{C} \longrightarrow \mathbb{C}^{n+1} \quad(0 \leq \ell \leq k),
$$

up to order $k$, and then take their wedge product

$$
\widetilde{F}_{k}:=\widetilde{f}^{(0)} \wedge \cdots \wedge \widetilde{f}^{(k)}: \mathbb{C} \longrightarrow \Lambda^{k+1}\left(\mathbb{C}^{n+1}\right) .
$$

Relating to the standard basis $\left\{e_{i}\right\}_{i=0, \ldots, n}$ of $\mathbb{C}^{n+1}$, by (6) there holds

$$
\widetilde{F}_{k}=\sum_{0 \leq i_{0}<i_{1}<\ldots<i_{k} \leq n} W\left(f_{i_{0}}, \cdots, f_{i_{k}}\right) e_{i_{0}} \wedge \cdots \wedge e_{i_{k}}
$$

in Plücker coordinates, where $W\left(f_{i_{0}}, \ldots, f_{i_{k}}\right):=\operatorname{det}\left(\left(f_{i_{\beta}}^{(\alpha)}\right)_{\alpha, \beta=0, \ldots, k}\right)$ is a standard Wronskian. For the purpose of descending the image of $\widetilde{F}_{k}$ along the natural projection

$$
\pi: \Lambda^{k+1}\left(\mathbb{C}^{n+1}\right) \backslash\{0\} \rightarrow \mathbb{P}\left(\Lambda^{k+1}\left(\mathbb{C}^{n+1}\right)\right)
$$

we now cancel the common zeros of all the obtained Wronskians by an auxiliary holomorphic function $g$ satisfying

$$
(g)_{0}=\min _{0 \leq i_{0}<i_{1}<\cdots<i_{k} \leq n}\left(W\left(f_{i_{0}}, \ldots, f_{i_{k}}\right)\right)_{0} .
$$

Hence the quotient succeeds

$$
\bar{F}_{k}:=\widetilde{F}_{k} / g: \mathbb{C} \longrightarrow \Lambda^{k+1}\left(\mathbb{C}^{n+1}\right) \backslash\{0\} .
$$

Definition 2.1. The $k^{\text {th }}$-derived curve of $f$ is

$$
F_{k}:=\pi \circ \bar{F}_{k}: \mathbb{C} \longrightarrow \mathbb{P}\left(\Lambda^{k+1}\left(\mathbb{C}^{n+1}\right)\right) .
$$


Recall that the Cartan's order function of $F_{k}$ is given by

$$
\begin{aligned}
T_{F_{k}}(r) & =\frac{1}{2 \pi} \int_{0}^{2 \pi} \log \left\|F_{k}\left(r e^{i \theta}\right)\right\|_{\max } \mathrm{d} \theta \\
& =\frac{1}{2 \pi} \int_{0}^{2 \pi} \log \max _{0 \leq i_{0}<i_{1}<\cdots<i_{k} \leq n}\left|\frac{W\left(f_{i_{0}}, \ldots, f_{i_{k}}\right)}{g}\left(r e^{i \theta}\right)\right| \mathrm{d} \theta .
\end{aligned}
$$

It is known that all the derived curves have the same growth rate (see e.g. [6]):

$$
T_{F_{k}}=O\left(T_{F_{\ell}}\right) \quad(0 \leq k, \ell \leq n) .
$$

A decomposable hyperplane

$$
A:=\pi\left\{Z \in \Lambda^{k+1}\left(\mathbb{C}^{n+1}\right): A^{*}(Z)=0\right\} \subset \mathbb{P}\left(\Lambda^{k+1}\left(\mathbb{C}^{n+1}\right)\right)
$$

is the dual of a nonzero decomposable $(k+1)$-vector

$$
A^{*}=a_{0} \wedge \cdots \wedge a_{k} \in \Lambda^{k+1}\left(\mathbb{C}^{n+1}\right)^{\vee} \cong\left(\Lambda^{k+1} \mathbb{C}^{n+1}\right)^{\vee} \quad\left(a_{0}, \ldots, a_{k} \in\left(\mathbb{C}^{n+1}\right)^{\vee}\right)
$$

We claim that the image of the derived curve $F_{k}(\mathbb{C})$ is not contained in any decomposable hyperplane. Indeed, for any $A^{*}$ above, writing each $a_{i}$ in the standard dual basis $\left\{e_{j}^{*}\right\}_{j=0, \ldots, n}$ of $\left(\mathbb{C}^{n+1}\right)^{\vee}$ as $a_{i}=\sum_{j=0}^{n} a_{i, j} e_{j}^{*}$, by formula (6) we have

$$
\begin{aligned}
A^{*}\left(\widetilde{F}_{k}\right) & =\sum_{0 \leq i_{0}<i_{1}<\ldots<i_{k} \leq n} \operatorname{det}\left(\left(a_{\alpha, i_{\beta}}\right)_{0 \leq \alpha, \beta \leq k}\right) e_{i_{0}}^{*} \wedge \cdots \wedge e_{i_{k}}^{*}\left(\widetilde{F}_{k}\right) \\
\text { [recall (9)] } & =\sum_{0 \leq i_{0}<i_{1}<\ldots<i_{k} \leq n} \operatorname{det}\left(\left(a_{\alpha, i_{\beta}}\right)_{0 \leq \alpha, \beta \leq k}\right) \operatorname{det}\left(\left(f_{i_{\beta}}^{(\alpha)}\right)_{0 \leq \alpha, \beta \leq k}\right) \\
\text { [by Cauchy-Binet Formula] } & =\operatorname{det}\left(\left(h_{\beta}^{(\alpha)}\right)_{0 \leq \alpha, \beta \leq k}\right),
\end{aligned}
$$

where each $h_{i}=\sum_{j=0}^{n} a_{i, j} f_{j}=a_{i} \circ \tilde{f}$ for $i=0, \ldots, k$. The linearly independence of $f_{0}, \ldots, f_{n}$ as well as that of $a_{0}, \ldots, a_{k}$ guarantee that $h_{0}, \ldots, h_{k}$ are also linearly independent, whence the Wronskian

$$
\operatorname{det}\left(\left(h_{\beta}^{(\alpha)}\right)_{0 \leq \alpha, \beta \leq k}\right) \not \equiv 0,
$$

i.e., $F_{k}(\mathbb{C})$ is not contained in the decomposable hyperplane $A$ defined by $A^{*}$.

Therefore, we define the $m$-truncated counting function of $F_{k}$ with respect to $A$ as

$$
N_{F_{k}}^{[m]}(r, A):=N^{[m]}\left(r,\left(A^{*} \circ \bar{F}_{k}\right)_{0}\right)
$$

The $m$-defect of $F_{k}$ with respect to $A$ is then defined by

$$
\delta_{F_{k}}^{[m]}(A):=\liminf _{r \rightarrow \infty}\left(1-\frac{N_{F_{k}}^{[m]}(r, A)}{T_{F_{k}}(r)}\right)
$$

which according to the First Main Theorem satisfies $0 \leq \delta_{F_{k}}^{[m]}(A) \leq 1$. 


\subsection{Nochka's weights}

Let $N \geq n$ be two positive integers. Let $H_{1}, \ldots, H_{q} \subset \mathbb{P}^{n}(\mathbb{C})$ be $q \geq N+1$ hyperplanes defined by the linear forms $h_{1}^{*}, \ldots, h_{q}^{*} \in\left(\mathbb{C}^{n+1}\right)^{\vee}$, respectively.

Conventions. Denote by $Q$ the index set $\{1, \ldots, q\}$. For a subset $R \subset Q$, denote by $|R|$ its cardinality and by $\operatorname{rank}(R)$ the dimension of the linear subspace of $\left(\mathbb{C}^{n+1}\right)^{\vee}$ generated by $\left\{h_{i}\right\}_{i \in R}$.

Definition 2.2. The family $\left\{H_{i}\right\}_{i=1, \ldots, q}$ is said to be in $N$-subgeneral position if any $N+1$ hyperplanes in this family have empty intersection. When $N=n$, this family is said to be in general position.

Remark 2.1. Keeping the assumptions as in the statement of Theorem 1.1 we may regard $f$ as a linearly non-degenerate curve $f: \mathbb{C} \rightarrow \mathbb{P}^{n}(\mathbb{C}) \hookrightarrow \mathbb{P}^{N}(\mathbb{C})$, which induces the derived curve

$$
F_{k}: \mathbb{C} \rightarrow \mathbb{P}^{\mathfrak{n}-1}(\mathbb{C}) \hookrightarrow \mathbb{P}^{\mathfrak{N}-1}(\mathbb{C}),
$$

where $\mathfrak{N}=\left(\begin{array}{l}N+1 \\ k+1\end{array}\right)$ and $\mathfrak{n}=\left(\begin{array}{l}n+1 \\ k+1\end{array}\right)$. Still using $A_{i}$ to denote the cut loci $A_{i} \cap \mathbb{P}^{\mathfrak{n}-1}(\mathbb{C})$, then $\left\{A_{i}\right\}_{i \in Q}$ is a family of $q$ hyperplanes in $(\mathfrak{N}-1)$-subgeneral position of $\mathbb{P}^{\mathfrak{n}-1}(\mathbb{C})$. Hence Theorem 1.1 is a direct consequence of the Main Theorem.

Here is the main tool in Nochka's resolution [8] of Cartan's conjecture.

Theorem 2.1. Let $\left\{H_{i}\right\}_{i=1, \ldots, q}$ be a family of $q \geq 2 N-n+1$ hyperplanes in $N$-subgeneral position of $\mathbb{P}^{n}(\mathbb{C})$. Then there exists a family of rational constants $\{\omega(i)\}_{i=1, \ldots, q}$ satisfying the following conditions:

i) $0 \leq \omega(i) \leq 1$ for all $i=1, \ldots, q$;

ii) set $\widetilde{\omega}:=\max _{1 \leq i \leq q} \omega(i)$, then

$$
\sum_{i=1}^{q} \omega(i)=\widetilde{\omega}(q-2 N+n-1)+n+1
$$

iii) $\frac{n+1}{2 N-n+1} \leq \widetilde{\omega} \leq \frac{n}{N}$

iv) if $R$ is a subset of $Q$ with $0<|R| \leq N+1$, then

$$
\sum_{i \in R} \omega(i) \leq \operatorname{rank}(R)
$$

The constants $\omega(j)$ are called Nochka's weights and $\widetilde{\omega}$ is called Nochka's constant of the family $\left\{H_{i}\right\}_{i=1, \ldots, q}$. They satisfy the following key property (c.f. [9, Lem. 4.1.17]).

Proposition 2.1. Let $\left\{H_{i}\right\}_{1 \leq i \leq q}$ be a family of $q \geq 2 N-n+1$ hyperplanes in $N$-subgeneral position of $\mathbb{P}^{n}(\mathbb{C})$. Let $a_{1}, \ldots, a_{q} \geq 1$ be arbitrary constants. If $R$ is a subset of $Q$ having cardinality

$$
0<|R| \leq N+1
$$

then there exist distinct indices $i_{1}, \ldots, i_{\mathrm{rank}(R)} \in R$ such that

$$
\operatorname{rank}\left(\left\{i_{1}, \ldots, i_{\operatorname{rank}(R)}\right\}\right)=\operatorname{rank}(R) \text { and } \prod_{i \in R} \alpha_{i}^{\omega(i)} \leq \prod_{k=1}^{\operatorname{rank}(R)} \alpha_{i_{k}} \text {. }
$$




\section{Proof of the Main Theorem}

\subsection{Notation and conventions}

Fix a reduced representation $\left[f_{0}: \cdots: f_{n}\right]$ of $f$. Denote by $Q=\{1, \ldots, q\}$. Assume that the decomposable hyperplanes $A_{1}, \ldots, A_{q}$ are defined by $A_{1}^{*}, \ldots, A_{q}^{*} \in \Lambda^{k+1}\left(\mathbb{C}^{n+1}\right)^{\vee}$, respectively. Let $\mathcal{S}$ be the set consisting of all subsets of $\{0, \ldots, n\}$ having cardinality $k+1$, which in the lexicography order writes as $\mathcal{S}=\left\{I_{0}, I_{1}, \ldots, I_{\mathfrak{n}-1}\right\}$. For every $I \in \mathcal{S}$, denote by $\|I\|$ its number of ranking, so that $\left\|I_{i}\right\|=i+1$ for $0 \leq i \leq \mathfrak{n}-1$. For $I, J \in \mathcal{S}$, denote by $W(I, J)$ the determinant of the matrix $\left(f_{j}^{(i)}\right)_{i \in I, j \in J}$. Hence $W\left(I_{0}, J\right)$ coincides with the usual Wronskian $W\left(\left\{f_{j}\right\}_{j \in J}\right)$. Let $\mathcal{W}=\left(W\left(I_{r}, I_{s}\right)\right)_{0 \leq r, s \leq \mathfrak{n}-1}$ be the $(k+1)^{\text {th }}$-compound matrix of $\left(f_{j}^{(i)}\right)_{0 \leq i, j \leq n}$. Then the SylvesterFranke theorem states that

$$
\operatorname{det} \mathcal{W}=W\left(f_{0}, \ldots, f_{n}\right)^{\left(\begin{array}{c}
n \\
k
\end{array}\right)}
$$

Hence the zero order of $\operatorname{det} \mathcal{W}$ is well-defined, invariant under coordinate changes. In fact, its estimation will be a major challenge in this paper, and we will use some elaborate coordinate system.

\subsection{An a priori estimate}

From now on, we assume that $q-2 \mathfrak{N}+\mathfrak{n}>0$, otherwise there is nothing to prove in the Main Theorem.

Let $\{\omega(i)\}_{i \in Q}$ be the Nochka's weights and let $\widetilde{\omega}$ be the Nochka's constant of the family $\left\{A_{i}\right\}_{i \in Q}$. Recalling the construction of the derived curve $F_{k}$, we first find some holomorphic function $g$ whose zero divisor is

$$
\mathcal{D}_{k}:=\min _{J \in \mathcal{S}}\left(W\left(I_{0}, J\right)\right)_{0}
$$

Here is an implement of Cartan's Wronskian technique and Nochka's estimate for derived curves.

Proposition 3.1. There exists some constant $C>0$ depending only on the family $\left\{A_{i}\right\}_{i \in Q}$ such that

$$
\begin{aligned}
\left\|F_{k}(z)\right\|_{\max }^{\widetilde{\omega}(q-2 \mathfrak{N}+\mathfrak{n})} \leq C \cdot\left(\frac{|g(z)|^{\mathfrak{n}} \prod_{i \in Q}\left|A_{i}^{*} \circ \bar{F}_{k}(z)\right|^{\omega(i)}}{|\operatorname{det}(\mathcal{W}(z))|}\right) \\
\times \sum_{R \subset Q, \operatorname{rank}(R)=|R|=\mathfrak{n}} \frac{|\operatorname{det}(\mathcal{W}(z))|}{\prod_{i \in R}\left|A_{i}^{*} \circ \widetilde{F}_{k}(z)\right|}
\end{aligned}
$$

Proof. The arguments follow closely to that of [9, page 125, Lem. 4.2.3]. Without loss of generality, we always assume that each hyperplane $A_{i}$ is defined by a linear form $A_{i}^{*}$ having unit norm $\left\|A_{i}^{*}\right\|=1$. Since $\left\{A_{i}\right\}_{i \in Q}$ is in $(\mathfrak{N}-1)$-subgeneral position, for any point $[Z] \in \mathbb{P}^{\mathfrak{n}-1}(\mathbb{C})$, where $Z \in \mathbb{C}^{\mathfrak{n}} \backslash\{0\}$, there is some index subset $S \subset Q$ with cardinality $|S|=q-\mathfrak{N}$ such that all the corresponding hyperplanes miss $[Z]$, namely $\prod_{i \in S} \frac{A_{i}^{*}(Z)}{\|Z\|} \neq 0$. Noting that $A_{i}^{*}(Z) /\|Z\|$ is well-defined for $[Z]$, by compactness argument, there exists some constant $C_{1}>0$ depending only on $\left\{A_{i}\right\}_{i \in Q}$ such that

$$
\frac{1}{C_{1}}<\sum_{S \subset Q,|S|=q-\mathfrak{N}} \prod_{i \in S}\left(\frac{\left|A_{i}^{*}(Z)\right|}{\|Z\|}\right)^{\omega(i)}<C_{1} \quad\left(\forall Z \in \mathbb{P}^{\mathfrak{n}-1}(\mathbb{C})\right) .
$$


Denote by $C(S)$ the complement of $S$ in $Q$. Now we can rewrite each term in the middle of the above inequality as

$$
\prod_{i \in S}\left(\frac{\left|A_{i}^{*}(Z)\right|}{\|Z\|}\right)^{\omega(i)}=\frac{\prod_{i \in Q}\left|A_{i}^{*}(Z)\right|^{\omega(i)}}{\|Z\|^{\sum_{i \in Q} \omega(i)}} \cdot \prod_{i \in C(S)}\left(\frac{\|Z\|}{\left|A_{i}^{*}(Z)\right|}\right)^{\omega(i)} .
$$

Since $\left\|A_{i}^{*}\right\|=1$, we have $\frac{\|Z\|}{\left|A_{i}^{*}(Z)\right|} \geq 1$. Noting that $C(S)$ has cardinality $\mathfrak{N}$, by the $(\mathfrak{N}-1)$-subgeneral assumption of $\left\{A_{i}\right\}_{i \in Q}$, we see that $\operatorname{rank}(C(S))=\mathfrak{n}$. Hence by Proposition 2.1, there exists an index subset $C_{0}(S) \subset C(S)$ having cardinality $\mathfrak{n}$ such that

$$
\prod_{i \in C(S)}\left(\frac{\|Z\|}{\left|A_{i}^{*}(Z)\right|}\right)^{\omega(i)} \leq \prod_{i \in C_{0}(S)} \frac{\|Z\|}{\left|A_{i}^{*}(Z)\right|} .
$$

Remembering that $\sum_{i \in Q} \omega(i)=\widetilde{\omega}(q-2 \mathfrak{N}+\mathfrak{n})+\mathfrak{n}$ by Theorem 2.1, we can estimate (17) as

$$
\begin{aligned}
\prod_{i \in S}\left(\frac{\left|A_{i}^{*}(Z)\right|}{\|Z\|}\right)^{\omega(i)} & \leq \frac{\prod_{i \in Q}\left|A_{i}^{*}(Z)\right|^{\omega(i)}}{\|Z\|^{\widetilde{\omega}(q-2 \mathfrak{N}+\mathfrak{n})+\mathfrak{n}}} \cdot \prod_{i \in C_{0}(S)} \frac{\|Z\|}{\left|A_{i}^{*}(Z)\right|} \\
& =\frac{\prod_{i \in Q}\left|A_{i}^{*}(Z)\right|^{\omega(i)}}{\|Z\|^{\widetilde{\omega}(q-2 \mathfrak{N}+\mathfrak{n})}} \cdot \frac{1}{\prod_{i \in C_{0}(S)}\left|A_{i}^{*}(Z)\right|}
\end{aligned}
$$

Taking the sum on both sides of the above inequality for all $S$ and using the lower bound of (16), we receive

$$
\|Z\|^{\widetilde{\omega}(q-2 \mathfrak{N}+\mathfrak{n})} \leq C_{1} \cdot\left(\prod_{i \in Q}\left|A_{i}^{*}(Z)\right|^{\omega(i)}\right) \cdot \sum_{S \subset Q,|S|=q-\mathfrak{N}} \frac{1}{\prod_{i \in C_{0}(S)}\left|A_{i}^{*}(Z)\right|}
$$

Substituting $Z$ by $\bar{F}_{k}(z)$ in the above inequality and noting that $\left\|F_{k}\right\|_{\max } \leq\left\|\bar{F}_{k}\right\|$, we receive

$$
\begin{aligned}
\left\|F_{k}(z)\right\|_{\max }^{\widetilde{\omega}(q-2 \mathfrak{N}+\mathfrak{n})} & \leq C_{1} \cdot\left(\prod_{i \in Q}\left|A_{i}^{*} \circ \bar{F}_{k}(z)\right|^{\omega(i)}\right) \cdot \sum_{S \subset Q,|S|=q-\mathfrak{N}} \frac{1}{\prod_{i \in C_{0}(S)}\left|A_{i}^{*} \circ \bar{F}_{k}(z)\right|} \\
& =C_{1} \cdot\left(\frac{\prod_{i \in Q}\left|A_{i}^{*} \circ \bar{F}_{k}(z)\right|^{\omega(i)}}{|\operatorname{det}(\mathcal{W}(z))|}\right) \times \sum_{S \subset Q,|S|=q-\mathfrak{N}} \frac{|\operatorname{det}(\mathcal{W}(z))||g(z)|^{\mathfrak{n}}}{\prod_{i \in C_{0}(S)}\left|A_{i}^{*} \circ \widetilde{F}_{k}(z)\right|} \\
& \leq C \cdot\left(\frac{|g(z)|^{\mathfrak{n}} \prod_{i \in Q}\left|A_{i}^{*} \circ \bar{F}_{k}(z)\right|^{\omega(i)}}{|\operatorname{det}(\mathcal{W}(z))|}\right) \times \sum_{R \subset Q,|R|=\operatorname{rank}(R)=\mathfrak{n}} \frac{|\operatorname{det}(\mathcal{W}(z))|}{\prod_{i \in R}\left|A_{i}^{*} \circ \widetilde{F}_{k}(z)\right|},
\end{aligned}
$$

whence concludes the proof.

\subsection{Fujimoto's vanishing order estimates}

In order to estimate the vanishing order of $\operatorname{det} \mathcal{W}$ effectively, Fujimoto [5, Section 5] employed the following nice coordinate system. The existence is essentially guaranteed by Gaussian elimination.

Lemma 3.1. Let $f: \mathbb{C} \rightarrow \mathbb{P}^{n}(\mathbb{C})$ be a linearly nondegenerate entire holomorphic curve. For a given point $z_{0} \in \mathbb{C}$, there exist some homogeneous coordinates of $\mathbb{P}^{n}(\mathbb{C})$, a reduced representation of $f$, and a local coordinate $z$ in a small neighborhood $U$ of $z_{0}$ such that $f$ can be written as $f=\left[f_{0}: \cdots: f_{n}\right]$, where

$$
f_{i}=z^{\alpha_{i}}+\sum_{j>\alpha_{i}} b_{i j} z^{j} \quad\left(b_{i j} \in \mathbb{C} ; 0 \leq i \leq n\right)
$$

on $U$ and $\alpha_{0}=0<\alpha_{1}<\cdots<\alpha_{n}$. 
Thus, he received the following estimates, assuming a nice coordinate system for (18).

Corollary 3.1. One has $\mathcal{D}_{k}\left(z_{0}\right)=\sum_{i=0}^{k}\left(\alpha_{i}-i\right)$.

Definition 3.1. The weight $\mathrm{w}(I)$ of a set $I=\left\{i_{0}, \ldots, i_{k}\right\}$ where $0 \leq i_{0}<i_{1}<\cdots<i_{k}<\infty$ is defined to be

$$
\mathrm{w}(I):=\left(i_{0}-0\right)+\cdots+\left(i_{k}-k\right) .
$$

Remark 3.1. If moreover $I \subset\{0,1, \ldots, n\}$, then one has

$$
\mathrm{w}(I) \leq \mathrm{w}(\{n-k, n-k+1, \ldots, n\})=(n-k)(k+1) .
$$

Corollary 3.2. For every $I, J \in S$, one has

$$
(W(I, J))_{0} \geq \sum_{a \in \mathbb{C}}\left(\mathcal{D}_{k}(a)-\mathrm{w}(I)+\mathrm{w}(J)\right)^{+}\{a\} .
$$

Running $I, J$ through $\mathcal{S}$, the summation of $-\mathrm{w}(I)$ and $\mathrm{w}(J)$ just cancel each other. Hence we obtain the following

Proposition 3.2. One has

$$
(\operatorname{det}(\mathcal{W}))_{0} \geq \mathfrak{n} \mathcal{D}_{k}
$$

Since both sides of the above inequality is independent of coordinates, it is in fact a general estimate.

\subsection{Fujimoto's trick}

Here is an essential ingredient in the proof of the key estimate (33) below.

Proposition 3.3 (Fujimoto). [5] Lem. 4.2] Let $h_{0}, \ldots, h_{k}$ be linearly independent meromorphic functions. Let $0 \leq i_{0}<i_{1}<\cdots<i_{k}$ be integers. Then the meromorphic function

$$
\frac{\operatorname{det}\left(h_{\ell}^{\left(i_{j}\right)}\right)_{j, \ell=0, \ldots, k}}{\operatorname{det}\left(h_{\ell}^{(i)}\right)_{i, \ell=0, \ldots, k}}
$$

can be written as a polynomial whose variables are of the form

$$
\left(\frac{\left(\operatorname{det}\left(h_{\ell_{i}}^{(j)}\right)_{i, j=0, \ldots, r}\right)^{\prime}}{\operatorname{det}\left(h_{\ell_{i}}^{(j)}\right)_{i, j=0, \ldots, r}}\right)^{(\lambda-1)}
$$

$\left(0 \leq r \leq k ; \lambda \geq 1 ; 0 \leq \ell_{0}<\ell_{1}<\cdots<\ell_{r} \leq k\right)$.

Furthermore, if one associates weight $\lambda$ with the above variable, then this polynomial can be chosen to be isobaric of weight $\mathrm{w}(I)$ where $I=\left\{i_{0}, \ldots, i_{k}\right\}$.

Corollary 3.3. For any point $a \in \mathbb{C}$, one has

$$
\operatorname{ord}_{a} \operatorname{det}\left(h_{\ell}^{\left(i_{j}\right)}\right)_{j, \ell=0, \ldots, k} \geq \operatorname{ord}_{a} \operatorname{det}\left(h_{\ell}^{(i)}\right)_{i, \ell=0, \ldots, k}-\mathrm{w}(I) .
$$

When $h_{1}, \ldots, h_{k}$ are holomorphic, we can provide an alternative interpretation of (20) as follows. Considering the holomorphic map

$$
\mathrm{h}=\left[h_{0}: \cdots: h_{k}\right]: \mathbb{C} \longrightarrow \mathbb{P}^{k}(\mathbb{C}),
$$

by Lemma 3.1, in a small neighborhood around $a \in \mathbb{C}$, we can change the homogeneous coordinates of $\mathbb{P}^{k}(\mathbb{C})$ by some invertible matrix $P$ such that the representation $\left(f_{0}, \ldots, f_{k}\right)=P\left(h_{0}, \ldots, h_{0}\right)$ of $\mathrm{h}$ in these coordinates satisfies the increasing vanishing shape of (18). Thus by taking $n=k, J=I_{0}$ in Corollary 3.2 we receive

$$
\operatorname{ord}_{a} \operatorname{det}\left(f_{\ell}^{\left(i_{j}\right)}\right)_{j, \ell=0, \ldots, k} \geq \operatorname{ord}_{a} \operatorname{det}\left(f_{\ell}^{(i)}\right)_{i, \ell=0, \ldots, k}-\mathrm{w}(I),
$$

which in exactly (20). 


\subsection{A vanishing order estimate}

Proposition 3.4. The following inequality holds:

$$
\sum_{i \in Q} \omega(i)\left(A_{i}^{*} \circ \bar{F}_{k}\right)_{0}-(\operatorname{det}(\mathcal{W}))_{0}+\mathfrak{n} \mathcal{D}_{k} \leq \sum_{i \in Q} \omega(i) \sum_{a \in \mathbb{C}} \min \left\{\operatorname{ord}_{a} A_{i}^{*} \circ \bar{F}_{k},(k+1)(n-k)\right\}\{a\} .
$$

Proof. The idea of the proof is to implement Nochka's weight technique [8] in the course of Fujimoto's vanishing order estimates [5, Prop. 5.3]. Since

$$
\operatorname{ord}_{a} A_{i}^{*} \circ \bar{F}_{k}=\min \left\{\operatorname{ord}_{a} A_{i}^{*} \circ \bar{F}_{k},(k+1)(n-k)\right\}+\left(\operatorname{ord}_{a} A_{i}^{*} \circ \bar{F}_{k}-(k+1)(n-k)\right)^{+},
$$

we can restate the inequality (21) as

$$
(\operatorname{det}(\mathcal{W}))_{0} \geq \mathfrak{n} \mathcal{D}_{k}+\sum_{i \in Q} \omega(i) \sum_{a \in \mathbb{C}}\left(\operatorname{ord}_{a} A_{i}^{*} \circ \bar{F}_{k}-(k+1)(n-k)\right)^{+} \cdot\{a\} .
$$

It is a pointwise inequality, hence for every fixed $a \in \mathbb{C}$ we focus on the indices

$$
S:=\left\{i \in Q: \operatorname{ord}_{a} A_{i}^{*} \circ \bar{F}_{k} \geq(k+1)(n-k)+1\right\},
$$

having nonzero contribution to the right hand side of (22). By Corollary 19, we only need to consider the case that $S \neq \emptyset$. Moreover, we claim that $|S|<\mathfrak{N}$. Indeed, suppose on the contrary that $S$ contains $\mathfrak{N}$ indices, say $1, \ldots, \mathfrak{N}$. By the assumption of subgeneral position, the corresponding hyperplanes $A_{1}, \ldots, A_{\mathfrak{N}}$ have empty intersection, hence at least one $A_{i}^{*} \circ \bar{F}_{k}(a) \neq 0$, contradicting to the selection of $S$.

Now we exhibit the distinct values $\left\{\operatorname{ord}_{a} A_{i}^{*} \circ \bar{F}_{k}\right\}_{i \in S}$ from high to low

$$
m_{1}>m_{2}>\cdots>\cdots>m_{t},
$$

and then set a filtration of $S$ accordingly $S_{0}:=\emptyset \subset S_{1} \subset \cdots \subset S_{t}=S$, where for every $i \in S_{\ell} \backslash S_{\ell-1}$, there holds $\operatorname{ord}_{a} A_{i}^{*} \circ \bar{F}_{k}=m_{\ell}$, respectively for $\ell=1, \ldots, t$. Let $\left\{T_{\ell} \subset S_{\ell}\right\}_{\ell=1, \ldots, t}$ be a family of increasing subsets $T_{1} \subset \cdots \subset T_{t}$ constructed subsequently by the law $\left|T_{\ell}\right|=\operatorname{rank}\left(T_{\ell}\right)=\operatorname{rank}\left(S_{\ell}\right)$. Set $\widetilde{m_{\ell}}=m_{\ell}-(k+1)(n-k)$. Now we can estimate

$$
\begin{aligned}
& \sum_{i \in Q} \omega(i)\left(\operatorname{ord}_{a} A_{i}^{*} \circ \bar{F}_{k}-(k+1)(n-k)\right)^{+} \\
= & \sum_{i \in S} \omega(i)\left(\operatorname{ord}_{a} A_{i}^{*} \circ \bar{F}_{k}-(k+1)(n-k)\right) \\
= & \sum_{\ell=1}^{t} \sum_{i \in S_{\ell} \backslash S_{\ell-1}} \omega(i) \widetilde{m_{\ell}} \\
= & \sum_{\ell=1}^{t}\left(\sum_{i \in S_{\ell}} \omega(i) \widetilde{m_{\ell}}-\sum_{j \in S_{\ell-1}} \omega(j) \widetilde{m_{\ell}}\right) \\
= & \left(\widetilde{m_{1}}-\widetilde{m_{2}}\right) \sum_{i \in S_{1}} \omega(i)+\left(\widetilde{m_{2}}-\widetilde{m_{3}}\right) \sum_{i \in S_{2}} \omega(i)+\cdots+\widetilde{m_{t}} \sum_{i \in S_{t}} \omega(i)
\end{aligned}
$$

[by (12)] $\leq\left(\widetilde{m_{1}}-\widetilde{m_{2}}\right) \operatorname{rank}\left(S_{1}\right)+\left(\widetilde{m_{2}}-\widetilde{m_{3}}\right) \operatorname{rank}\left(S_{2}\right)+\cdots+\widetilde{m_{t}} \operatorname{rank}\left(S_{t}\right)$

$$
\begin{aligned}
& =\operatorname{rank}\left(S_{1}\right) \widetilde{m_{1}}+\left(\operatorname{rank}\left(S_{2}\right)-\operatorname{rank}\left(S_{1}\right)\right) \widetilde{m_{2}}+\cdots+\left(\operatorname{rank}\left(S_{t}\right)-\operatorname{rank}\left(S_{t-1}\right)\right) \widetilde{m_{t}} \\
& =\left|T_{1}\right| \widetilde{m_{1}}+\left(\left|T_{2}\right|-\left|T_{1}\right|\right) \widetilde{m_{2}}+\cdots+\left(\left|T_{t}\right|-T_{t-1} \mid\right) \widetilde{m_{t}}
\end{aligned}
$$

$=\left|T_{1}\right| \widetilde{m_{1}}+\left|T_{2} \backslash T_{1}\right| \widetilde{m_{2}}+\cdots+\left|T_{t} \backslash T_{t-1}\right| \widetilde{m_{t}}$. 
Changing the indices of hyperplanes $\left\{A_{i}\right\}_{i \in Q}$ if necessary, we may assume that $T_{t}=\left\{1, \ldots,\left|T_{t}\right|\right\}$. Set $m_{s}^{*}=\operatorname{ord}_{a} A_{s}^{*} \circ \bar{F}_{k}$ for $s=1, \ldots,\left|T_{t}\right|$. Then (23) reads as

$$
\sum_{i \in Q} \omega(i)\left(\operatorname{ord}_{a} A_{i}^{*} \circ \bar{F}_{k}-(k+1)(n-k)\right)^{+} \leq \sum_{s=1}^{\left|T_{t}\right|}\left(m_{s}^{*}-(k+1)(n-k)\right) .
$$

Hence the desired inequality (22) can be established by showing a stronger estimate

$$
\operatorname{ord}_{a} \operatorname{det}(\mathcal{W}) \geq \mathfrak{n} \mathcal{D}_{k}(a)+\sum_{s=1}^{\left|T_{t}\right|}\left(m_{s}^{*}-(k+1)(n-k)\right) .
$$

We first recall that a similar a priori estimate (19) can be achieved by applying Lemma 3.1, Indeed, we can calculate the vanishing orders of $\operatorname{det}(\mathcal{W})$ and $\mathcal{D}_{k}$ at the given point $a$ more effectively by means of nice coordinates, in which $f_{0}, \ldots, f_{n}$ have explicit increasing vanishing shapes as (18). Thus for every $I, J \in \mathcal{S}$ the $(\|I\|,\|J\|)$-th entry of $\mathcal{W}$ has vanishing order $\geq \mathcal{D}_{k}-\mathrm{w}(I)+\mathrm{w}(J)$, whence det $\mathcal{W}$ satisfies the estimate (19) by straightforward summation based on the Laplace expansion. But to reach the stronger estimate (25) we need more effort, inevitably by exploiting the extra condition that $\left\{A_{j}^{*} \circ \bar{F}_{k}\right\}_{j=1, \ldots,\left|T_{t}\right|}$ have high vanishing orders. Here is our strategy. We will modify some $\left|T_{t}\right|$ columns of $\mathcal{W}$ to represent the information of $\left\{A_{j}^{*} \circ \bar{F}_{k}\right\}_{j=1, \ldots,\left|T_{t}\right|}$, by multiplying certain wellchosen invertible matrix I. Thus the new obtained matrix $\widetilde{\mathcal{W}}=\mathcal{W} \cdot \mid$ keeps the same vanishing order of determinant. Now for $s=1, \ldots,\left|T_{t}\right|$ the $s$-th "new column" of $\widetilde{\mathcal{W}}$ contribute, in each entry, at least $m_{s}^{*}-(k+1)(n-k)$ more vanishing order estimate than that of $\mathcal{W}$. Whence by counting vanishing order in each term of the Laplacian expansion of $\operatorname{det}(\widetilde{\mathcal{W}})_{0}$, we conclude the proof.

Now we carry out the details. Starting with the following

Fact. Let $\left\{v_{i}\right\}_{i=1, \ldots, m}$ be a basis of a linear space $V$, and let $\widetilde{v}_{1}, \ldots, \widetilde{v}_{\ell} \in V$ be some linearly independent vectors. Then one can replace some $\ell$ vectors in $\left\{v_{i}\right\}_{i=1, \ldots, m}$ by $\widetilde{v}_{1}, \ldots, \widetilde{v}_{\ell}$ such that they still form a basis.

Applying the above fact to $V=\Lambda^{k+1}\left(\mathbb{C}^{n+1}\right)^{\vee}$ and its basis $\left\{e_{I_{i}}^{*}=\Lambda_{\ell \in I_{i}} e_{\ell}^{*}\right\}_{i=0,1, \ldots, \mathfrak{n}-1}$, we can replace some $\left|T_{t}\right|$ vectors $e_{I_{i_{1}}}^{*}, \ldots, e_{I_{\left|T_{t}\right|}}^{*}$ by $A_{1}^{*}, \ldots, A_{\left|T_{t}\right|}^{*}$ respectively to receive a new basis

$$
\left(b_{1}, \ldots, b_{\mathfrak{n}}\right)=\left(e_{I_{0}}^{*}, \ldots, e_{I_{\mathfrak{n}-1}}^{*}\right) \cdot \mathbf{l}
$$

where according to our construction, I differs from the identity matrix only in the columns $i_{1}+$ $1, \ldots, i_{\left|T_{t}\right|}+1$.

Write $A_{1}^{*}=l_{0} \wedge \cdots \wedge l_{k}$, where linear forms $l_{j} \in\left(\mathbb{C}^{n+1}\right)^{\vee}$ comparing to the standard basis $\left\{e_{j}^{*}\right\}_{j=0, \ldots, n}$ read as $\left(l_{0}, \ldots, l_{k}\right)=\left(e_{0}^{*}, \ldots, e_{n}^{*}\right) \cdot L$ for some $(n+1) \times(k+1)$ matrix $L$. By (6) we have

$$
A_{1}^{*}=\sum_{0 \leq j_{0}<\cdots<j_{k} \leq n} \operatorname{det}\left(L_{\left\{j_{0}, \ldots, j_{k}\right\}}\right) e_{j_{0}}^{*} \wedge \cdots \wedge e_{j_{k}}^{*}=\sum_{i=0}^{\mathfrak{n}-1} \operatorname{det}\left(L_{I_{i}}\right) e_{I_{i}}^{*},
$$

where $L_{\left\{j_{0}, \ldots, j_{k}\right\}}$ consists of the rows $j_{0}+1, \ldots, j_{k}+1$ of $L$. This shows all the entries of the $\left(i_{1}+1\right)$-th column of $\mathrm{I}$, and hence the $\left(\|J\|, i_{1}+1\right)$-th entry of $\widetilde{\mathcal{W}}=\mathcal{W} \cdot \mathrm{I}$ is nothing but:

$$
\sum_{i=0}^{\mathfrak{n}-1} \operatorname{det}\left(L_{I_{i}}\right) W\left(J, I_{i}\right)=\sum_{I \in \mathcal{S}} \operatorname{det}\left(L_{I}\right) \operatorname{det}\left(\left(f_{\ell}^{(j)}\right)_{j \in J, \ell \in I}\right)
$$

[by Cauchy-Binet Formula] $=\operatorname{det}\left(\left(h_{\ell}^{(j)}\right)_{j \in J, \ell=0, \ldots, k}\right)$, 
where similar to (11) we have

$$
\left(h_{0}, \ldots, h_{k}\right)=\left(l_{0} \circ \tilde{f}, \ldots, l_{k} \circ \tilde{f}\right)
$$

for the lifting $\widetilde{f}$ given in (7). Noting that $h_{\ell}^{(j)}=l_{\ell} \circ \widetilde{f}^{(j)}$, setting $\widetilde{F}^{J}:=\wedge_{j \in J} \widetilde{f}^{(j)}$, then (29) becomes

$$
\begin{aligned}
\operatorname{det}\left(\left(h_{\ell}^{(j)}\right)_{j \in J, \ell=0, \ldots, k}\right) & =\operatorname{det}\left(\left(l_{\ell} \circ \widetilde{f}^{(j)}\right)_{j \in J, \ell=0, \ldots, k}\right) \\
& =\left(l_{0} \wedge \cdots \wedge l_{k}\right) \cdot\left(\wedge_{j \in J} \widetilde{f}^{(j)}\right) \\
& =A_{1}^{*} \circ \widetilde{F}^{J} .
\end{aligned}
$$

In particular, for $J=I_{0}=\{0, \ldots, k\}$, the $\left(1, i_{1}+1\right)$-th entry of $\widetilde{\mathcal{W}}$ is $A_{1}^{*} \circ \widetilde{F}_{k}=g \cdot\left(A_{1}^{*} \circ \bar{F}_{k}\right)$, which is known to have high vanishing order $\mathcal{D}_{k}(a)+m_{1}^{*}$ at the point $a$. Lastly, applying Corollary 3.3 upon the neat determinant (29) and using Remark 3.1, we conclude that for any $J \in \mathcal{S}$ the $\left(\|J\|, i_{1}+1\right)$-th entry of $\widetilde{\mathcal{W}}$ has vanishing order at least

$$
\mathcal{D}_{k}(a)+m_{1}^{*}-\mathrm{w}(J) \geq\left(\mathcal{D}_{k}(a)-\mathrm{w}(J)+\mathrm{w}\left(I_{i_{1}}\right)\right)+\left(m_{1}^{*}-(k+1)(n-k)\right) .
$$

Similarly, for $s=1, \ldots,\left|T_{t}\right|$, the same argument shows that the $\left(\|J\|, i_{s}+1\right)$-th entry of $\widetilde{\mathcal{W}}$ has vanishing order at least :

$$
\mathcal{D}_{k}(a)+m_{s}^{*}-\mathrm{w}(J) \geq\left(\mathcal{D}_{k}(a)-\mathrm{w}(J)+\mathrm{w}\left(I_{i_{s}}\right)\right)+\left(m_{s}^{*}-(k+1)(n-k)\right) .
$$

Note that the first bracket above is exactly the original vanishing order estimate of the $\left(\|J\|, i_{s}+1\right)$-th entry of $\mathcal{W}$, and that the second bracket is a summand in (25). By straightforward summation based on the Laplace expansion, we conclude the proof.

\subsection{An application of the logarithmic derivative lemma}

Here is an estimate due to Fujimoto [5].

Proposition 3.5. One has the estimate

$$
\frac{1}{2 \pi} \int_{0}^{2 \pi} \max _{R \subset Q,|R|=\operatorname{rank}(R)=\mathfrak{n}} \log \frac{|\operatorname{det}(\mathcal{W})|}{\prod_{i \in R}\left|A_{i}^{*} \circ \widetilde{F}_{k}\right|}\left(r e^{i \theta}\right) \mathrm{d} \theta=S_{F_{k}}(r) .
$$

For the sake of completeness, we include a proof here. To start with, let us recall

Logarithmic derivative Lemma. [9] Lem. 4.2.9] Let $g$ be a nonconstant meromorphic function on $\mathbb{C}$. Then for any integer $\ell \geq 1$, the following estimate holds

$$
m_{\left(\frac{g^{\prime}}{g}\right)}^{(\ell)}(r):=m_{\left(\frac{g^{\prime}}{g}\right)}^{(\ell)}(r)(r, \infty)=S_{g}(r) .
$$

To prove (33), one must get rid of $g$ in the left-hand side. Hence it is necessary to work in logarithmic setting. Taking the wedge products of the logarithmic derivatives

$$
f_{\log }^{(\ell)}=\left(\left(\frac{f_{0}}{f_{0}}\right)^{(\ell)}, \ldots,\left(\frac{f_{n}}{f_{0}}\right)^{\ell)}\right): \mathbb{C} \longrightarrow \mathbb{C}^{n+1} \quad(\ell=0,1, \ldots, k),
$$

we obtain the logarithmic derived curve

$$
\widetilde{F}_{k, \log }=f_{\log }^{(0)} \wedge \cdots \wedge f_{\log }^{(k)}: \mathbb{C} \longrightarrow \Lambda^{k+1} \mathbb{C}^{n+1},
$$


which in Plücker coordinates reads as

$$
\widetilde{F}_{k, \log }=\sum_{0 \leq i_{0}<i_{1}<\cdots<i_{k} \leq n} W_{\log }\left(f_{i_{0}}, \ldots, f_{i_{k}}\right) e_{i_{0}} \wedge \cdots \wedge e_{i_{k}},
$$

where

$$
W_{\log }\left(f_{i_{0}}, \ldots, f_{i_{k}}\right):=\operatorname{det}\left(\left(f_{i_{\beta}} / f_{0}\right)^{(\alpha)}\right)_{\alpha, \beta=0, \ldots, k}=f_{0}^{-(k+1)} W\left(f_{i_{0}}, \ldots, f_{i_{k}}\right)
$$

is the logarithmic Wronskian. Hence we have

$$
\widetilde{F}_{k, \log }=f_{0}^{-(k+1)} \widetilde{F}_{k} .
$$

For $I, J \in \mathcal{S}$, the logarithmic analog $W_{\log }(I, J)$ of $W_{I, J}$ is defined to be the determinant of the matrix $\left(\left(f_{j} / f_{0}\right)^{(i)}\right)_{i \in I, j \in J}$. Setting

$$
\mathcal{W}_{\log }:=\left(W_{\log }\left(I_{r}, I_{s}\right)\right)_{0 \leq r, s \leq \mathfrak{n}-1},
$$

by Sylvester-Franke theorem we have

$$
\begin{aligned}
\operatorname{det}\left(\mathcal{W}_{\log }\right) & =W_{\log }\left(f_{0}, \ldots, f_{n}\right)\left(\begin{array}{c}
n \\
k
\end{array}\right) \\
& =\left(f_{0}^{-(n+1)} W\left(f_{0}, \ldots, f_{n}\right)\right)^{\left(\begin{array}{c}
n \\
k
\end{array}\right)} \\
{[\text { recall (13) }] } & =f_{0}^{-(k+1) \mathfrak{n}} \operatorname{det}(\mathcal{W}),
\end{aligned}
$$

where in the last equality we need a straightforward calculation $(n+1)\left(\begin{array}{l}n \\ k\end{array}\right)=(k+1)\left(\begin{array}{c}n+1 \\ k+1\end{array}\right)=(k+1) \mathfrak{n}$.

Proof of Proposition 3.5. By (34), (35), we rewrite

$$
\frac{|\operatorname{det}(\mathcal{W})|}{\prod_{i \in R}\left|A_{i}^{*} \circ \widetilde{F}_{k}\right|}=\frac{\left|\operatorname{det}\left(\mathcal{W}_{\log }\right)\right|}{\prod_{i \in R}\left|A_{i}^{*} \circ \widetilde{F}_{k, \log }\right|} .
$$

Hence

$$
\max _{R \subset Q,|R|=\operatorname{rank}(R)=\mathfrak{n}} \log \frac{|\operatorname{det}(\mathcal{W})|}{\prod_{i \in R}\left|A_{i}^{*} \circ \widetilde{F}_{k}\right|} \leq \sum_{R \subset Q,|R|=\operatorname{rank}(R)=\mathfrak{n}} \log ^{+} \frac{\left|\operatorname{det}\left(\mathcal{W}_{\log }\right)\right|}{\prod_{i \in R}\left|A_{i}^{*} \circ \widetilde{F}_{k, \log }\right|} .
$$

We now analyze each summand above. Without loss of generality, we illustrate by $R=\{1, \ldots, \mathfrak{n}\}$ for simplicity of indices. Since $\left\{A_{i}^{*}\right\}_{i \in R}$ form a basis for $\Lambda^{k+1}\left(\mathbb{C}^{n+1}\right)^{\vee}$, changing coordinates we read

$$
\left(A_{1}^{*}, \ldots, A_{\mathfrak{n}}^{*}\right)=\left(e_{I_{0}}^{*}, \ldots, e_{I_{\mathfrak{n}-1}}^{*}\right) \cdot \mathrm{C}
$$

for an invertible $\mathfrak{n} \times \mathfrak{n}$-matrix C. Now the matrix $\widetilde{\mathcal{W}}_{\log }:=\mathcal{W}_{\log } \cdot \mathrm{C}$, similar to $\widetilde{\mathcal{W}}$ below (26), has a neat expression of determinant in each entry. Indeed, setting $\widetilde{F}_{\log }^{I}:=\wedge_{i \in I} f_{\log }^{(i)}$ for every $I \in \mathcal{S}$, by the same argument as (28), (29), the $(|I|, j)$-th entry of $\widetilde{\mathcal{W}}_{\text {log }}$ is nothing but $A_{j}^{*} \circ \widetilde{F}_{\log }^{I}$. Thus

$$
\begin{aligned}
\frac{\left|\operatorname{det}\left(\mathcal{W}_{\log }\right)\right|}{\prod_{i \in R}\left|A_{i}^{*} \circ \widetilde{F}_{k, \log }\right|} & =\frac{\left|\operatorname{det}\left(\widetilde{\mathcal{W}}_{\log } \cdot \mathrm{C}^{-1}\right)\right|}{\prod_{i=1, \ldots, \mathfrak{n}}\left|A_{i}^{*} \circ \widetilde{F}_{k, \log }\right|} \\
& =\left|\operatorname{det}\left(\mathrm{C}^{-1}\right)\right| \times \frac{\left|\operatorname{det}\left(A_{j}^{*} \circ \widetilde{F}_{\log }^{I_{i-1}}\right)_{i, j=1, \ldots, \mathfrak{n}}\right|}{\prod_{j=1, \ldots, \mathfrak{n}}\left|A_{j}^{*} \circ \widetilde{F}_{k, \log }\right|} \\
& =\left|\operatorname{det}\left(\mathrm{C}^{-1}\right)\right| \times\left|\operatorname{det}\left(\frac{A_{j}^{*} \circ \widetilde{F}_{\log }^{I_{i-1}}}{A_{j}^{*} \circ \widetilde{F}_{k, \log }}\right)_{i, j=1, \ldots, \mathfrak{n}}\right| .
\end{aligned}
$$


Using the basic inequalities

$$
\log ^{+}\left(\sum_{i=1}^{p} x_{i}\right) \leq \sum_{i=1}^{p} \log ^{+} x_{i}+\log p, \quad \log ^{+}\left(\prod_{i=1}^{p} x_{i}\right) \leq \sum_{i=1}^{p} \log ^{+} x_{i}
$$

we have

$$
\log ^{+} \frac{\left|\operatorname{det}\left(\mathcal{W}_{\log }\right)\right|}{\prod_{i \in R}\left|A_{i}^{*} \circ \widetilde{F}_{k, \log }\right|} \leq \sum_{i, j=1, \ldots, \mathfrak{n}} \log ^{+}\left|\frac{A_{j}^{*} \circ \widetilde{F}_{\log }^{I_{i-1}}}{A_{j}^{*} \circ \widetilde{F}_{k, \log }}\right|+C,
$$

where $C$ is some constant independent of $f$. Now the problem reduces to showing that

$$
\frac{1}{2 \pi} \int_{0}^{2 \pi} \log ^{+}\left|\frac{A_{j}^{*} \circ \widetilde{F}_{\log }^{I}}{A_{j}^{*} \circ \widetilde{F}_{k, \log }}\right|\left(r e^{i \theta}\right) \mathrm{d} \theta=S_{F_{k}}(r)
$$

for any $I \in \mathcal{S}$ and $j \in Q$. We illustrate by $j=1$ and $A_{1}^{*}=a_{0} \wedge \cdots \wedge a_{k}$, where each $a_{i} \in\left(\mathbb{C}^{n+1}\right)^{\vee}$ in the standard dual basis $\left\{e_{j}^{*}\right\}_{j=0, \ldots, n}$ reads as $a_{i}=\sum_{j=0}^{n} a_{i, j} e_{j}^{*}$. Similar to (29), we have

$$
A_{j}^{*} \circ \widetilde{F}_{\log }^{I}=\operatorname{det}\left(\left(h_{\ell}^{(i)}\right)_{i \in I, \ell=0, \ldots, k}\right)
$$

where each $h_{\ell}=\sum_{i=0}^{n} a_{\ell, i} f_{i} / f_{0}$. Now by applying Proposition 3.3 and the Logarithmic Derivative Lemma, the desired estimate (40) follows directly from (10), (38) and the following

Fact. [9, page. 78, Thm. 2.5.13] For every $i=0,1, \ldots, n$, one has the estimate

$$
T\left(r, f_{i} / f_{0}\right) \leq O\left(T_{f}(r)\right) .
$$

\subsection{End of the proof of the Main Theorem}

Taking logarithm on both sides of (15) and then integrating, we receive

$$
\widetilde{\omega}(q-2 \mathfrak{N}+\mathfrak{n}) T_{F_{k}}(r) \leq \frac{1}{2 \pi} \int_{0}^{2 \pi} \log \varphi\left(r e^{i \theta}\right) \mathrm{d} \theta+\frac{1}{2 \pi} \int_{0}^{2 \pi} \psi\left(r e^{i \theta}\right) \mathrm{d} \theta+O(1),
$$

where

$$
\varphi=\frac{|g|^{\mathfrak{n}} \prod_{i \in Q}\left|A_{i}^{*} \circ \bar{F}_{k}\right|^{\omega(i)}}{|\operatorname{det}(\mathcal{W})|}, \quad \psi=\sum_{R \subset Q, \operatorname{rank}(R)=|R|=\mathfrak{n}} \frac{|\operatorname{det}(\mathcal{W})|}{\prod_{i \in R}\left|A_{i}^{*} \circ \widetilde{F}_{k}\right|} .
$$

Using Proposition 3.4, we receive

$$
\begin{aligned}
(\varphi)_{0} & =\sum_{i \in Q} \omega_{i}\left(A_{i}^{*} \circ \bar{F}_{k}\right)_{0}+\mathfrak{n}\left(\mathcal{D}_{k}\right)_{0}-(\operatorname{det} \mathcal{W})_{0} \\
& \leq \sum_{i \in Q} \omega(i) \sum_{a \in \mathbb{C}} \min \left\{\operatorname{ord}_{a} A_{i}^{*} \circ \bar{F}_{k},(k+1)(n-k)\right\}\{a\} \\
& \leq \widetilde{\omega} \sum_{i \in Q} \sum_{a \in \mathbb{C}} \min \left\{\operatorname{ord}_{a} A_{i}^{*} \circ \bar{F}_{k},(k+1)(n-k)\right\}\{a\} .
\end{aligned}
$$

Whence by Jensen formula we have

$$
\begin{aligned}
\frac{1}{2 \pi} \int_{0}^{2 \pi} \log \left|\varphi\left(r e^{i \theta}\right)\right| \mathrm{d} \theta & \leq N_{\varphi}(r, 0)+O(1) \\
& \leq \widetilde{\omega} \sum_{i \in Q} N_{F_{k}}^{[(k+1)(n-k)]}\left(r, A_{i}\right)+O(1) .
\end{aligned}
$$

Together this with (41) and Proposition 3.5, we finish the proof. 


\section{Some applications}

\subsection{A defect relation}

Defect relation. Let $f: \mathbb{C} \rightarrow \mathbb{P}^{n}(\mathbb{C})$ be a linearly nondegenerate entire holomorphic curve. For a fixed integer $k=0,1, \ldots, n$, let $A_{1}, \ldots, A_{q} \subset \mathbb{P}\left(\Lambda^{k+1}\left(\mathbb{C}^{n+1}\right)\right)$ be $q$ decomposable hyperplanes such that any $\mathfrak{N}$ of them have empty intersection. Then the $k$-th derived curve $F_{k}$ of $f$ satisfy the following estimate:

$$
\sum_{i=1}^{q} \delta_{F_{k}}^{[(k+1)(n-k)]}\left(A_{i}\right) \leq 2 \mathfrak{N}-\mathfrak{n} .
$$

Proof. The Main Theorem can be rewritten as

$$
\sum_{i=1}^{q}\left(1-\frac{N_{F_{k}}^{[(k+1)(n-k)]}\left(r, A_{i}\right)}{T_{F_{k}}(r)}\right) \leq 2 \mathfrak{N}-\mathfrak{n}+\frac{S_{F_{k}}(r)}{T_{F_{k}}(r)}
$$

Taking the limit inferior of both sides of the above inequality, we conclude the proof.

\subsection{Ramification Theorem}

Theorem 4.1. In the setting of the Main Theorem, assuming moreover that the associated $k$-th derived curve $F_{k}$ is completely $\mu_{k, i}$-ramified over each decomposable hyperplane $A_{i}$ for $i=1, \ldots, q$, then one has

$$
\sum_{i=1}^{q}\left(1-\frac{(k+1)(n-k)}{\mu_{k, i}}\right) \leq 2 \mathfrak{N}-\mathfrak{n} .
$$

Proof. For an index $i$ with $\mu_{k, i}<\infty$, every nonzero coefficients of the divisor $\left(A_{i}^{*} \circ \bar{F}_{k}\right)_{0}$ is $\geq \mu_{i}^{k}$. Hence

$$
\begin{aligned}
& \delta_{F_{k}}^{[(k+1)(n-k)]}\left(A_{i}\right)=1-\lim \sup \frac{N_{F_{k}}^{[(k+1)(n-k)]}\left(r, A_{i}\right)}{T_{F_{k}}(r)} \\
& \geq 1-(k+1)(n-k) \limsup \frac{N_{F_{k}}^{[1]}\left(r, A_{i}\right)}{T_{F_{k}}(r)} \\
& \text { [By the First Main Theorem] } \\
& \geq 1-(k+1)(n-k) \limsup \frac{N_{F_{k}}^{[1]}\left(r, A_{i}\right)}{N_{F_{k}}\left(r, A_{i}\right)} \\
& \geq 1-\frac{(k+1)(n-k)}{\mu_{i}^{k}} .
\end{aligned}
$$

When $\mu_{i}^{k}=\infty$, the above inequality is trivial. By the defect relation we finish the proof.

\section{References}

[1] Lars V. Ahlfors. The theory of meromorphic curves. Acta Soc. Sci. Fennicae. Nova Ser. A., 3(4):31, 1941.

[2] Henri Cartan. Sur les zéros des combinaisons linéarires de $p$ fonctions holomorphes données. Mathematica, 7:5-31, 1933. 
[3] Wan Xi Chen. Defect relations for degenerate meromorphic maps. Trans. Amer. Math. Soc., 319(2):499-515, 1990.

[4] Michael Cowen and Phillip Griffiths. Holomorphic curves and metrics of negative curvature. $J$. Analyse Math., 29:93-153, 1976.

[5] Hirotaka Fujimoto. The defect relations for the derived curves of a holomorphic curve in $P^{n}(\mathbf{C})$. Tohoku Math. J. (2), 34(1):141-160, 1982.

[6] Hirotaka Fujimoto. Value distribution theory of the Gauss map of minimal surfaces in $\mathbf{R}^{m}$. Aspects of Mathematics, E21. Friedr. Vieweg \& Sohn, Braunschweig, 1993.

[7] Rolf Nevanlinna. Zur Theorie der Meromorphen Funktionen. Acta Math., 46(1-2):1-99, 1925.

[8] E. I. Nochka. On the theory of meromorphic curves. Dokl. Akad. Nauk SSSR, 269(3):547-552, 1983.

[9] Junjiro Noguchi and Jörg Winkelmann. Nevanlinna theory in several complex variables and Diophantine approximation, volume 350 of Grundlehren der Mathematischen Wissenschaften [Fundamental Principles of Mathematical Sciences]. Springer, Tokyo, 2014.

[10] Min Ru. Holomorphic curves into algebraic varieties. Ann. of Math. (2), 169(1):255-267, 2009.

[11] Min Ru. A Cartan's second main theorem approach in Nevanlinna theory. Acta Math. Sin. (Engl. Ser.), 34(8):1208-1224, 2018.

[12] B. V. Shabat. Distribution of values of holomorphic mappings, volume 61 of Translations of Mathematical Monographs. American Mathematical Society, Providence, RI, 1985. Translated from the Russian by J. R. King, Translation edited by Lev J. Leifman.

[13] Wilhelm Stoll. Die beiden Hauptsätze der Wertverteilungstheorie bei Funktionen mehrerer komplexer Veränderlichen. I. Acta Math., 90:1-115, 1953.

[14] Wolhelm Stoll. Die beiden Hauptsätze der Wertverteilungstheorie bei Funktionen mehrerer komplexer Veränderlichen. II. Acta Math., 92:55-169, 1954.

[15] Hermann Weyl. Meromorphic Functions and Analytic Curves. Annals of Mathematics Studies, no. 12. Princeton University Press, Princeton, N. J., 1943.

[16] Hermann Weyl and Joachim Weyl. Meromorphic curves. Ann. of Math. (2), 39(3):516-538, 1938.

[17] Hung-Hsi Wu. The equidistribution theory of holomorphic curves. Annals of Mathematics Studies, No. 64. Princeton University Press, Princeton, N.J.; University of Tokyo Press, Tokyo, 1970 .

Dinh Tuan Huynh, Hua Loo-Keng Center for Mathematical Sciences, Academy of Mathematics and System Science, Chinese Academy of Sciences, Beijing 100190, China \& Department of Mathematics, University of Education, Hue University, 34 Le Loi St., Hue City, Vietnam

E-mail address: dinh-tuan.huynheamss.ac.cn

Song-Yan Xie, Academy of Mathematics and System Science \& Hua Loo-Keng Key Laboratory of Mathematics, Chinese ACADEmy of Sciences, BeiJing 100190, China

E-mail address: xiesongyan@amss.ac.cn 